\title{
Keratopathy due to ophthalmic drug abuse with corneal melting and perforation presenting as Mooren-like ulcer: A case report
}

\author{
HUI WU ${ }^{1}$, YUAN HU ${ }^{2}$, XIAO-RU SHI ${ }^{1}$, FEIHONG XU ${ }^{2}$, CHUN-YING JIANG $^{1}$, RONG HUANG $^{1}$ and HUI JIA ${ }^{1}$ \\ ${ }^{1}$ Department of Ophthalmology, First Affiliated Hospital of Jilin University, Changchun, Jilin 130021, P.R. China; \\ ${ }^{2}$ Department of Pathology, Mount Sinai School of Medicine, New York, NY 10029, USA
}

Received December 24, 2014; Accepted February 11, 2016

DOI: $10.3892 /$ etm.2016.3296

\begin{abstract}
The present report describes the clinical course and treatment of a Mooren-like ulcer associated with abuse of topical anesthetics and dexamethasone. A 38-year-old male physician treated himself with lidocaine, tetracaine and dexamethasone (DEX) eye drops for severe pain and decline of vision in both eyes. After six months of treatment, his right cornea exhibited annular melting with full-thickness stromal infiltration at the limbus and central corneal haze. His left cornea was completely melted and exhibited iris prolapse. The patient was treated with topical antibiotics, lubricants and underwent a binocular keratoplasty. The surgery was successful and after eight months the postoperative best-corrected visual acuities were counting fingers in the right eye and light perception in the left eye. In summary, this report documents a severe case of keratopathy presenting as Mooren-like ulcer caused by topical anesthetics and DEX, which were treated via keratoplasty, resulting in useful vision being retained.
\end{abstract}

\section{Introduction}

Topical ocular anesthetics are typically safe when used appropriately. However, their widespread use has revealed a potential to cause severe toxicity, abuse and addiction. Keratopathy is a common manifestation of topical ophthalmic drug abuse, and results in intense pain, loss of vision, refractory central corneal epithelial defects, ring keratitis, degenerative keratopathy with epithelial cell shedding, stromal infiltration, and may lead to corneal melting and perforation, with poor prognosis $(1,2)$. Abuse of topical ocular anesthetics is rare and serious toxicities

Correspondence to: Professor Hui Jia, Department of Ophthalmology, First Affiliated Hospital of Jilin University, 71 Xinmin Street, Changchun, Jilin 130021, P.R. China

E-mail: jiahui560515@163.com

Abbreviations: BCVA, best corrected visual acuity; DEX, dexamethasone

Key words: keratopathy, anesthetic abuse, dexamethasone, keratoplasty are not frequently reported. In numerous cases, the symptoms of toxic keratopathy are mistaken for another disease. Mooren's ulcer is an idiopathic, chronic, non-infectious, painful ulcerative keratitis with a characteristic undermined opaque central edge. The ulcerative lesion with overhanging edges typically initially presents on the periphery of the cornea and often spreads progressively to the entire circumference or towards the centre of the cornea $(3,4)$. The disease may also be recurrent in nature and involve one or both eyes. The common manifestations of topical ophthalmic drug abuse keratopathy include intense pain, loss of vision, refractory central corneal epithelial defects, ring keratitis, degenerative keratopathy with epithelial cell shedding, stromal infiltration, and may lead to corneal melting and perforation, with poor prognosis $(1,2)$. Here we report a typical case of toxic keratopathy presented as binocular corneal annular melt and perforation, subsequent to the abuse of lidocaine, tetracaine and dexamethasone (DEX) eye drops, the manifestations of which are similar to those of Mooren's ulcer.

\section{Case report}

The patient was a 38-year-old male physician with severe pain and decline of vision in the left and right eyes for six months. He had treated himself with sodium hyaluronate eye drops (Santen Pharmaceutical Co., Ltd., Shenzhen, China), lidocaine eye drops (Tianjin Jin Yao Amino Acid Co., Ltd., Tianjin, China) and DEX eye drops (Shandong Xinhua Pharmaceutical Co., Ltd., Henan, China) hourly for the previous six months. However, the deterioration of his eyes continued and the patient began to self-administer tetracaine eye drops (Zhejiang Jiuxu Pharmaceutical Co., Ltd., Hangzhou, China) once per hour during the two weeks prior to being admitted to the Department of Ophthalmology at First Hospital of Jilin University (Changchun, China). Written informed consent was obtained from the patient for publication of this case report and accompanying images. The present study was approved by the Human Ethics Committee of Jilin University, (Changchun, China) and written informed consent was obtained from the patient.

The patient had a history of intermittent use of lidocaine and DEX eye drops in both eyes ten years previously. His best-corrected visual acuities (BCVAs) (5) at admission were $20 / 32$ in the right eye and hand motion in the left eye. 

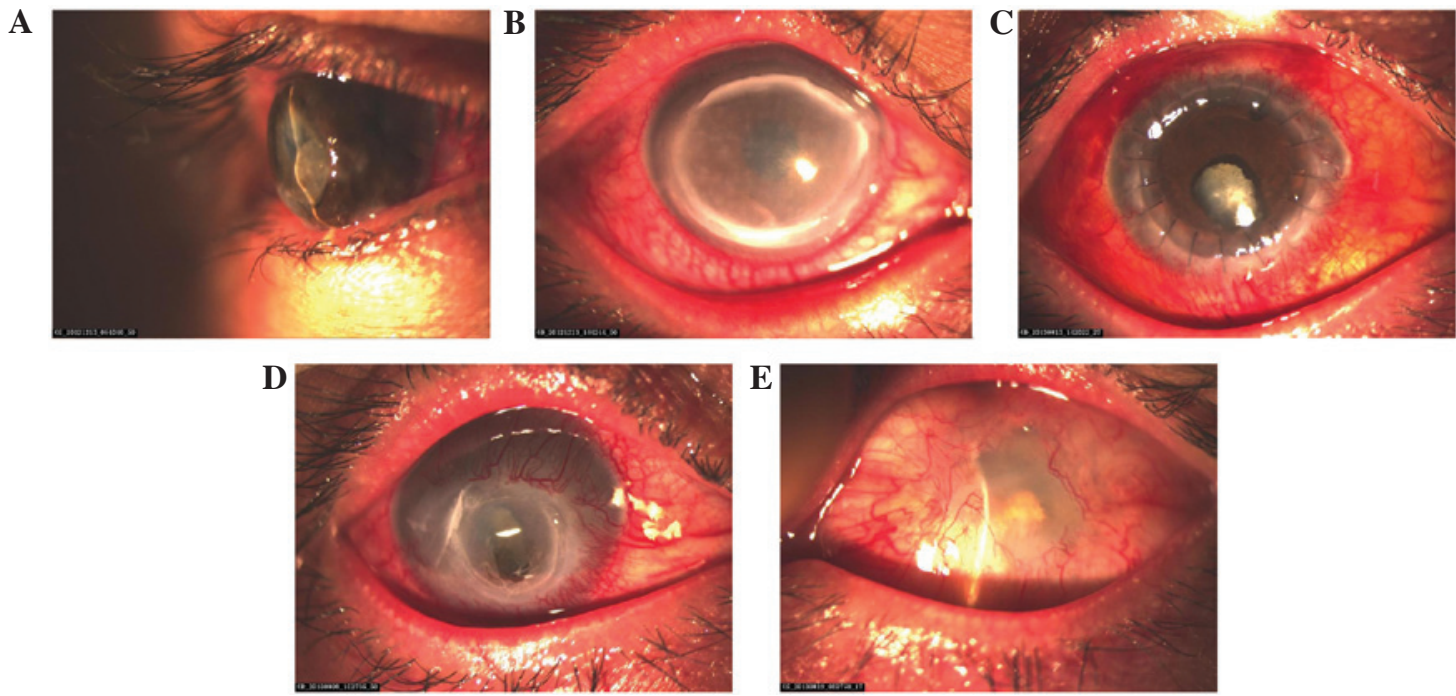

Figure 1. Slit lamp images of both eyes prior to and following surgery. (A) Right eye at first admission: An annular corneal thinning 2-2.5 mm to the limbus is shown and spots of gray opacity in the central cornea. (B) Left eye at first admission: A thinning of the whole cornea with a perforation $\sim 2.5 \times 2 \mathrm{~mm}$ filled with iris on the central cornea, and the anterior chamber disappeared. (C) Right eye at second admission: The cornea was gray and opaque, thin and with neovascularization. There was a perforation $\sim 5 \times 4 \mathrm{~mm}$ filled with iris at the bottom of the central cornea, and the anterior chamber disappeared. (D) Right eye after the surgery: The graft was transparent and in location with a normal anterior chamber. (E) Left eye, 8-months postsurgery: Proliferated bulbar conjunctiva and new vessels covered most of the corneal tissue.
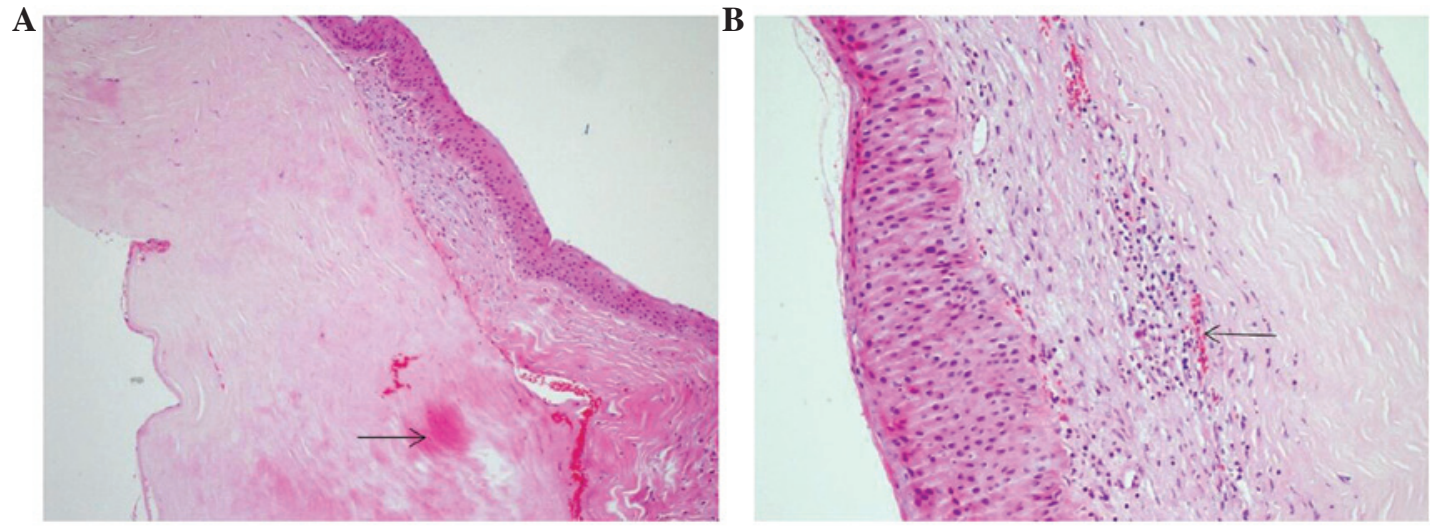

Figure 2. Histological sections of the right cornea stained with hematoxylin and eosin. (A) Infiltration of a large number of neutrophils, lymphocytes, and plasma cells in the cornea. The arrow shows a large area of typical hyaline degeneration in the corneal stroma (magnification, x100). (B) Hyperplasia and neovascularization of the corneal epithelium. The arrow indicates infiltration of a large number of neutrophils, lymphocytes and plasma cells in the cornea (magnification, $\mathrm{x} 200$ ).

His right cornea exhibited annular melting with Mooren-like full-thickness stromal infiltration at the limbus; central corneal haze was also observed (Fig. 1A). His left cornea was completely melted and iris prolapse was observed (Fig. 1B).

Use of the anesthetics and DEX eye drops was stopped and an emergency keratoplasty was performed in his left eye immediately following admission. The homogeneous allogeneic corneal tissue was provided by the eye bank of the First Affiliated Hospital of Jilin University. Following the surgery, the left eye was treated with 4 drops per eye per day (one drop administered on four separate occasions) $0.05 \%$ cyclosporine eye drops (North China Pharmaceutical Co., Ltd., Shijiazhuang, China), $0.5 \%$ levofloxacin eye drops and $0.1 \%$ sodium hyaluronate eye drops (Santen Pharmaceutical Co., Ltd.) six times daily per eye, with one drop administered on each occasion, and $100 \%$ autologous serum eye drops three times daily per eye, administered as one drop on three separate occasions. Eleven days postsurgery, the patient's BCVA of the left eye was light perception. No corneal allograft rejection was observed.

After release from the hospital, the patient did not comply with follow-up. Eight months later, he presented again with redness and loss of vision in the right eye of $\sim 10$ days duration. The patient had self-administered tobramycin and DEX ointment (Alcon; Novartis International AG, Basel, Switzerland) three times per day since his release from the hospital, and used lidocaine eye drops again to control pain. The patient was re-admitted to the hospital and his BCVA was hand motion in the right eye. His right cornea was gray and thin. A perforation $\sim 3 \times 2 \mathrm{~mm}$ in size and iris prolapse at the inferior of the central cornea was observed; the anterior chamber had disappeared (Fig. 1C).

A penetrating keratoplasty was immediately performed in his right eye; the homogeneous allogeneic corneal tissue was provided by our eye bank. After the surgery, the right eye was 
treated with tacrolimus eye drops (Zhongshan Ophthalmic Center, Zhongshan, China) and fluorometholone eye drops four times daily, sodium hyaluronate eye drops (Santen Pharmaceutical Co., Ltd.) six times daily, and atropine sulfate eye gel (Shenyang Xing Qi Pharmaceutical Co., Ltd., Shenyang, China) three times daily.

Prior to hospital discharge, the patient's BCVAs included counting fingers with use of the right eye and light perception in the left eye. There was no allograft rejection (Fig. 1D), and the bulbar conjunctiva of the left eye proliferated and covered the majority of the cornea (Fig. 1E).

Hematoxylin and eosin staining was performed as follows: Dewaxing formalin-fixed samples with xylene for $10 \mathrm{~min}$, followed by further dewaxing with xylene for $5 \mathrm{~min}$. Next, samples underwent two washes with absolute alcohol for $1 \mathrm{~min}$ each, followed by 3 successive washes with 95,90 and then $85 \%$ alcohol, each for $1 \mathrm{~min}$. Subsequently, samples were washed with distilled tap water for $2 \mathrm{~min}$, stained with hematoxylin for 1-5 min, washed with distilled tap water for $1 \mathrm{~min}$, then with $1 \%$ diluted ammonia water for $30 \mathrm{sec}$, and again with distilled water for $1 \mathrm{~min}$. Next, staining with eosin for $20 \mathrm{sec}$ to $5 \mathrm{~min}$, washing with distilled water for $30 \mathrm{sec}$, then successive rinses with 85, 90 and 95\% alcohol for $20 \mathrm{sec}, 30 \mathrm{sec}$ and $1 \mathrm{~min}$, respectively. Further washes with $95 \%$ alcohol for $1 \mathrm{~min}$, and absolute alcohol twice each for $2 \mathrm{~min}$. Finally, samples were treated with xylene three times, each for 2 min. Next, samples were fixed in rhamsan gum (neutral resin) sealing piece and cut into $2 \times 2 \mathrm{~cm}$ sections $4 \mu \mathrm{m}$ thick. Sections were then vieswed under an Olympus BX50 microscope with magnification, x50. Histology of the resection of the right cornea revealed corneal epithelial hyperplasia, neovascularization, hyaline degeneration of the corneal stroma, with infiltration of neutrophils, lymphocytes and plasma cells (Fig. 2A and B).

\section{Discussion}

In general, Mooren's ulcer is an idiopathic, crescent-shaped peripheral corneal ulcer with grey white infiltrate. The characteristic lesion begins in the periphery of the cornea and spreads circumferentially (6). The leading edges of the ulcer are undermined, infiltrated and de-epithelialized. In the present case, we assume that the application of topical anesthetics altered the antigens of a normal cornea and led to an autoimmune response. Anesthetic-induced keratopathy is progressive and is accompanied by a injury of the corneal epithelium (7-9). Early corneal damage can be identified on the first day of topical application of an anesthetic, and is characterized as a smoothly structured periphery and a pitted corneal surface. If not treated or improperly treated for a number of days or weeks, infiltration in the corneal stroma may appear along the rims of the area of defected epithelium, forming a progressive ring lesion that can lead to corneal ulceration, thinning and eventually perforation and endophthalmitis $(10,11)$. Prompt diagnosis is crucial, as patients typically continue to use topical anesthetics for pain management if no warning is given.

The mechanism underlying toxic keratopathy may include damage of the corneal epithelial barrier and disruption of re-epithelialization (6). A healthy epithelial surface protects the cornea from potential infection and the invasion of various microbial pathogens. Topical anesthetics may paralyze corneal perception, reduce blink reflex and suppress reflex weeping. This increases the duration of exposure of the cornea to the air and promotes apoptosis (10), and therefore the ability of corneal epithelial cells to resist infection and foreign bodies is significantly reduced. In addition to initially compromising the integrity of the corneal epithelial barrier, topical anesthetics exert inhibitory effects on respiration, glucose metabolism and mitosis of corneal epithelial cells, disrupting the process of re-epithelialization $(6,12,13)$. With continued use of topical anesthetics, eventually the corneal tissue may undergo necrosis, dissolution and perforation. Topical anesthetics may also alter corneal antigens and lead to an autoimmune response (14). The increased immune activity and inflammatory cells further damage the cornea, leading to a cycle of altered corneal antigens and self-destruction (15-17).

DEX is an anti-inflammatory medication with low corneal epithelial permeability. Excessive instillation of DEX eye drops may lead to various complications (18), including glaucoma, optic nerve damage, visual disturbance, sub-capsular cataract and corneal thinning (19-21), and significantly delays corneal re-epithelialization (22).

The prompt diagnosis and treatment of toxic keratopathy is crucial to the clinical outcome. However, diagnosis of keratopathy due to drug abuse is often delayed as the clinical manifestations are common to other diseases such as acanthamoeba keratitis, bacterial keratitis, viral keratitis and single cell virus keratitis $(23,24)$. Furthermore, the diagnosis may be complicated if the patient cannot accurately identify the eye drops they have used (25). A thorough history and consideration of the specific eye drops used are crucial for the accurate diagnosis of drug-induced keratopathy (26).

\section{References}

1. Ansari H, Garibaldi DC and Jun AS: Anaesthetic abuse keratopathy as a manifestation of ocular Munchausen's syndrome. Clin Experiment Ophthalmol 34: 81-83, 2006.

2. Yagci A, Bozkurt B, Egrilmez S, Palamar M, Ozturk BT and Pekel H: Topical anesthetic abuse keratopathy: A commonly overlooked health care problem. Cornea 30: 571-575, 2011.

3. Shinomiya K, Ueta M, Sotozono C, Inatomi T, Yokoi N, Koizumi N and Kinoshita S: Immunohistochemical analysis of inflammatory limbal conjunctiva adjacent to Mooren's ulcer. Br J Ophthalmol 97: 362-366, 2013.

4. Ngan ND and Chau HT: Amniotic membrane transplantation for Mooren's ulcer. Clin Experiment Ophthalmol 39: 386-392, 2011.

5. Murthy GVS, Johnson G: Chapter 1. In: The Epidemiology of Eye Disease. Johnson GJ, Minassian DC, Weale RA and West SK (eds). Vol 58. 3rd edition. World Scientific Publishing Company, Singapore, pp8, 2012.

6. Chang YS, Tseng SY, Tseng SH and Wu CL: Cytotoxicity of lidocaine or bupivacaine on corneal endothelial cells in a rabbit model. Cornea 25: 590-596, 2006.

7. Tok OY, Tok L, Atay IM, Argun TC, Demirci N and Gunes A: Toxic keratopathy associated with abuse of topical anesthetics and amniotic membrane transplantation for treatment. Int $\mathrm{J}$ Ophthalmol 8: 938-944, 2015.

8. Dass BA, Soong HK and Lee B: Effects of proparacaine on actin cytoskeleton of corneal epithelium. J Ocul Pharmacol 4: 187-194, 1988

9. Burns RP: Toxic effects of local anesthetics. JAMA 240: 347, 1978.

10. McGee HT and Fraunfelder FW: Toxicities of topical ophthalmic anesthetics. Expert Opin Drug Saf 6: 637-640, 2007.

11. Patel M and Fraunfelder FW: Toxicity of topical ophthalmic anesthetics. Expert Opin Drug Metab Toxicol 9: 983-988, 2013.

12. Atilla H, Tekeli O, Can B, Karel F and Saran Y: Effects of intracameral lidocaine on ocular tissues. Clin Experiment Ophthalmol 31: 73-77, 2003. 
13. Catterall WA: Molecular mechanisms of gating and drug block of sodium channels. Novartis Found Symp 241: 206-218; discussion 218-232, 2002.

14. Khakshoor H, Moshirfar M, Simpson RG, Gharaee H, Vejdani AH, Christiansen SM, Edmonds JN and Behunin NL: Anesthetic keratopathy presenting as bilateral Mooren-like ulcers. Clin Ophthalmol 6: 1719-1722, 2012.

15. Chen HT, Chen KH and Hsu WM: Toxic keratopathy associated with abuse of low dose anesthetic: A case report. Cornea 23: 527-529, 2004.

16. Kintner JC, Grossniklaus HE, Lass JH and Jacobs G: Infectious crystalline keratopathy associated with topical anesthetic abuse. Cornea 9: 77-80, 1990.

17. Meyers-Elliott RH, Pettit TH and Maxwell WA: Viral antigens in the immune ring of Herpes simplex stromal keratitis. Arch Ophthalmol 98: 897-904, 1980.

18. Swaminathan S, Vavia PR, Trotta F and Cavalli R: Nanosponges encapsulating dexamethasone for ocular delivery: Formulation design, physicochemical characterization, safety and corneal permeability assessment. J Biomed Nanotechnol 9: 998-1007, 2013.

19. Ferrante P, Ramsey A, Bunce $C$ and Lightman S: Clinical trial to compare efficacy and side-effects of injection of posterior sub-Tenon triamcinolone versus orbital floor methylprednisolone in the management of posterior uveitis. Clin Experiment Ophthalmol 32: $563-568,2004$
20. Zhang JZ, Krenzer KL, López FJ and Ward KW: Comparative effects of besifloxacin and other fluoroquinolones on corneal reepithelialization in the rabbit. J Cataract Refract Surg 36: 1049-1050, 2010.

21. Li CC and Chauhan A: Modeling ophthalmic drug delivery by soaked contact lenses. Ind Eng Chem Res 45: 3718-3734, 2006.

22. Piper SL, Laron D, Manzano G, Pattnaik T, Liu X, Kim HT and Feeley BT: A comparison of lidocaine, ropivacaine and dexamethasone toxicity on bovine tenocytes in culture. J Bone Joint Surg Br 94: 856-862, 2012.

23. Ardjomand N, Faschinger C, Haller-Schober EM, Scarpatetti $\mathrm{M}$ and Faulborn J: A clinico-pathological case report of necrotizing ulcerating keratopathy due to topical anaesthetic abuse. Ophthalmologe 99: 872-875, 2002 (In German).

24. Rosenwasser GO, Holland S, Pflugfelder SC, Lugo M, Heidemann DG, Culbertson WW and Kattan H: Topical anesthetic abuse. Ophthalmology 97: 967-972, 1990.

25. Katsimpris JM, Sarantoulakou M, Kordelou A, Petkou D and Petropoulos IK: Clinical findings in patients with topical anaesthetic abuse keratitis: A report of five cases. Klin Monbl Augenheilkd 224: 303-308, 2007.

26. Lee JK and Stark WJ: Anesthetic keratopathy after photorefractive keratectomy. J Cataract Refract Surg 34: 1803-1805, 2008. 